\title{
Radioprotection: Quelles innovations? Quel avenir ? Retour sur la première journée scientifique du Club des Jeunes Sociétaires de la SFRP
}

\author{
V. Ménard ${ }^{1, *}$, M. Maître ${ }^{2}$, M. Chambrette ${ }^{3}$, B.-M. Ayadi ${ }^{4}$, T. Beaumont ${ }^{4}$ et L.-A. Beltrami ${ }^{2}$ \\ ${ }^{1}$ CEA, DRF / Jacob/iRCM, Plateforme d'irradiation, B.P. 6, 18, route du panorama, 92265 Fontenay-Aux-Roses Cedex, France. \\ 2 CEPN, 28, rue de la redoute, 92260 Fontenay-aux-Roses, France. \\ 3 ENSAE, 5, Avenue Le Chatelier, 91120 Palaiseau, France. \\ ${ }^{4}$ IRSN, B.P. 17, 92262 Fontenay-aux-Roses Cedex, France.
}

Reçu le 11 septembre 2019 / Accepté le 25 octobre 2019

\begin{abstract}
Résumé - Le Club des Jeunes Sociétaires de la Société Française de Radioprotection a organisé sa première journée scientifique en avril 2018, avec pour thème: «Radioprotection: Quelles innovations? Quel avenir?». Réunissant plus d'une cinquantaine de participants, cette première journée s'est déroulée en deux temps avec des présentations plénières le matin et des ateliers de réflexion l'après-midi portant sur la manière dont l'innovation peut contribuer à la progression ainsi qu'à l'avenir de la radioprotection. Cet article présente les principaux éléments ressortis lors des discussions de ces deux ateliers. Les échanges ont mis en avant la nécessité de prendre en compte le développement des nouvelles technologies et d'en étudier leurs possibles applications aux champs de la radioprotection, que ce soit dans le domaine médical ou de la surveillance environnementale. Mais au-delà, les participants ont plus particulièrement insisté sur le besoin de déployer des outils innovants se plaçant au service de la promotion d'une culture de radioprotection et d'un meilleur partage des données. Pour ces deux enjeux, différentes actions ont été proposées et constituent ainsi des pistes d'actions dont le Club des Jeunes Sociétaires pourrait s'emparer à l'avenir.
\end{abstract}

Mots clés : radioprotection / innovation / futur / public / travailleurs / jeunes professionnels

\begin{abstract}
Radiation protection: What innovations? What future? Feedback on the first scientific day of the Young Club of the French Society for Radiation Protection. The "Young Club" of the French Society for Radiation Protection organized its first conference in April 2018, under the theme of: "Radiation protection: What innovations? What future?". Bringing together more than fifty participants, this first conference was organized in two stages with (i) plenary presentations in the morning and (ii) two workshops on how innovation can contribute to the future of radiation protection in the afternoon. This article aims to present the main elements highlighted during the discussions of these two workshops. Discussions raised indeed the need to consider the development of new technologies and to analyse their possible applications in the fields of radiation protection, whether in the medical field or environmental monitoring. And beyond that, participants also emphasized the need to set up innovative tools which could contribute to the promotion of a radiation protection culture and a better sharing of data. Regarding these key issues, various actions have been suggested and could be deemed as potential future actions for the "Young Club".
\end{abstract}

Keywords: radioprotection / innovation / future / public / workers / young professionals

\section{Introduction}

Le Club des Jeunes Sociétaires de la Société Française de Radioprotection (SFRP) regroupe des jeunes professionnels venant de tous horizons et motivés pour échanger sur les thématiques de radioprotection et promouvoir ce domaine.

\footnotetext{
$\overline{* A u t e u r}$ de correspondance $:$ veronique.menard@cea.fr
}

Une des missions premières du Club des Jeunes Sociétaires est de faciliter les échanges entre jeunes professionnels de la radioprotection, de favoriser la montée en compétence par l'organisation de journées thématiques, ainsi que de développer un réseau à l'échelle nationale et internationale. Une première journée scientifique a été organisée le 16 avril 2018 à la Maison des Associations de Solidarité à Paris, avec pour thème: «Radioprotection: Quelles innovations? Quel avenir?». 
L'objectif de cette journée, ouverte à tout public, a été de réunir des étudiants, doctorants, jeunes professionnels et professionnels confirmés pour réfléchir à la manière dont l'innovation peut s'inscrire et contribuer à la progression ainsi qu'à l'avenir de la radioprotection.

Organisée en deux temps, cette journée scientifique s'est partagée entre des présentations plénières le matin et des ateliers de réflexion en sous-groupes l'après-midi. En ce qui concerne la matinée, diverses communications orales ont été présentées. Elles concernaient à la fois les développements scientifiques mis en œuvre dans le domaine médical ainsi que les nouvelles technologies développées au service des professionnels et du public. Michel Bourguignon (IRSN), orateur invité, a d'ailleurs proposé en introduction de cette journée scientifique une réflexion sur l'avenir de la radioprotection, sur la base de son article : «Radioprotection : Quel avenir?» (Bourguignon et al., 2017).

L'après-midi, deux ateliers de réflexion ont été organisés : l'un sur le thème de l'innovation en radioprotection au service $\mathrm{du}$ public et l'autre sur le thème de l'innovation en radioprotection au service des travailleurs. Pour chacun de ces ateliers, chaque participant a pu nourrir la réflexion et apporter son point de vue en identifiant notamment les enjeux d'avenir pour la radioprotection du public ou des travailleurs, leurs possibles mises en œuvre ainsi que leurs limites et les risques associés.

Dans ce contexte, cet article a pour objectif de faire ressortir les principaux éléments issus de cette première journée scientifique et plus particulièrement des discussions ayant eu lieu lors des deux ateliers de réflexion.

\section{Méthodologie employée}

Afin de favoriser l'émergence d'une vision commune des enjeux d'avenir de la radioprotection pour le public ou les travailleurs, les ateliers de réflexion ont été animés en appliquant une technique de visualisation et de structuration de débat pour collecter les idées et construire une position collégiale.

Les deux ateliers ont été séquencés en quatre étapes, notamment pour :

- identifier les enjeux associés à l'innovation dans la radioprotection;

- proposer et partager des réponses ou des visions individuelles;

- analyser de manière collégiale les positions de chacun préalablement regroupées par thématiques;

- proposer des actions à mener et une synthèse commune.

Afin que chaque participant aux ateliers puisse avoir la possibilité d'exprimer ses idées, l'identification des enjeux a été effectuée à l'aide de «post-it» avec comme consigne : «un post-it, une idée». Les propositions ont été regroupées par thématique et priorisées par ordre d'intérêt de l'ensemble des participants. Ces derniers ont ensuite proposé des réponses appropriées et partagé leur vision, et ce, toujours à l'aide de «post-it». Compte-tenu du temps imparti, seuls les principaux thèmes ayant un intérêt majeur pour les participants ont été traités. Ensuite, l'analyse collégiale a d'abord porté sur la pertinence des réponses proposées vis-à-vis des enjeux identifiés, puis sur le rapport «bénéfice/risque » inhérent à toute innovation.

Pour finir, l'objectif de la dernière séquence de l'atelier a été de faire émerger des recommandations ou des actions qui pourraient être menées par le Club des Jeunes Sociétaires dans le cadre de ses activités.

\section{Retour sur les discussions issues des deux ateliers de réflexion}

Les échanges lors des deux ateliers de réflexion ont permis d'identifier plusieurs thèmes d'intérêt pour les années à venir. Les différents participants ont d'emblée soulevé la nécessité de prendre en compte le développement des nouvelles technologies (big data, open data, applications smartphone, etc.), d'en étudier leur possible application aux champs de la radioprotection et de voir dans quelle mesure ils pourraient être placés au service de la population et des travailleurs.

La surveillance de la radioactivité dans l'environnement a été donnée comme exemple de thématique pour laquelle les nouvelles technologies pourraient être utiles en cherchant notamment à développer des outils de détection plus fiables, plus performants et plus facilement accessibles au grand public.

$\mathrm{Au}$ cours des discussions dans les deux ateliers, les participants ont également tenu à souligner que pour ce qui concerne le domaine médical en particulier, des progrès sont attendus sur l'optimisation générale des méthodes de radiothérapie ainsi que sur le développement de thérapies innovantes et de procédés post-traitements visant à limiter les effets secondaires des radiothérapies. La poursuite des études sur la radiosensibilité individuelle, sur l'effet des faibles doses ainsi que l'amélioration des codes de calcul associés aux doses délivrées par les actes médicaux sont d'autres enjeux d'intérêt qui ont émergé lors des discussions. Une veille sur les résultats des projets de recherche européens en cours HARMONIC ${ }^{1}$, MEDIRAD $^{2}$ et VERIDIC $^{3}$ serait d'ailleurs intéressante à réaliser. Le développement d'une approche holistique de gestion des risques, cherchant à développer une vision intégrée des risques et, tenant compte de l'ensemble des contaminants environnementaux (dont les rayonnements ionisants), est aussi un challenge mis en avant par les différents participants.

En plus de ces enjeux, ces deux ateliers de réflexion ont plus particulièrement fait ressortir deux thématiques pour lesquelles l'innovation pourrait venir en appui. Ces thématiques concernent la promotion d'une culture de radioprotection et le besoin d'un meilleur partage des données.

\footnotetext{
${ }^{1}$ Health effects of cArdiac fluoRoscopy and mOdern radIotherapy in paediatriCs - https://www.isglobal.org/en/-/harmonic.

${ }^{2}$ The MEDIRAD Project aims to enhance the scientific bases and clinical practice of radiation protection in the medical field and thereby addresses the need to better understand and evaluate the health effects of low-dose ionising radiation exposure from diagnostic and therapeutic imaging and from off-target effects in radiotherapy. www.medirad-project.eu.

${ }^{3}$ Validation and Estimation of Radiation skIn Dose in Interventional Cardiology - https://www.researchgate.net/project/VERIDIC-Valida tion-and-Estimation-of-Radiation-skIn-Dose-in-Interventional-Cardi ology.
} 


\subsection{L'innovation au service de la promotion d'une culture de radioprotection}

Les discussions issues des ateliers ont fait valoir la nécessité de promouvoir une culture pratique de radioprotection aussi bien auprès des professionnels qu'auprès du grand public. En ce qui concerne la culture de radioprotection auprès des professionnels, elle peut parfois s'adresser à des personnes dont la radioprotection n'est pas le cœur de métier, mais qui vont devoir en appliquer les règles de manière à mener à bien leurs missions (e.g. personnel soignant). Par ailleurs, les participants ont pris l'exemple des Personnes Compétentes en Radioprotection $(\mathrm{PCR})^{4}$, qui, sorties d'école et nouvellement embauchées se sentent souvent esseulées et livrées à elles-mêmes. Le challenge est alors d'accompagner ces personnes dans la réalisation de leurs premières missions. Le «compagnonnage» par un «mentor» plus expérimenté pourrait être une piste de travail à explorer. En effet, cela pourrait aider la jeune PCR à acquérir de la rigueur dans l'exercice de ses fonctions d'une part, ainsi qu'à gagner en confiance en étant moins seule face aux différentes tâches demandées, d'autre part. Ce «compagnonnage» permettrait également d'assurer un transfert générationnel de compétences et de connaissances. Le domaine de la radioprotection et sa législation évoluant en permanence, le « compagnonnage» permettrait aussi au «mentor» de se mettre à jour des évolutions techniques et réglementaires.

Les participants ont également insisté sur l'emploi des nouveaux outils de formation à destination de l'ensemble des professionnels de la radioprotection. En effet, des méthodes de Serious Game, des outils de simulation ou encore des outils de mise en situation permettent aujourd'hui, en plus du savoir théorique, d'accéder à une mise en pratique virtuelle. Selon les participants, ces méthodes innovantes représentent une réelle valeur ajoutée pour permettre aux professionnels en devenir de se rendre compte des situations auxquelles ils vont être confrontés par la suite. Cependant, les discussions révèlent également certains écueils associés à l'utilisation de tels outils. Par exemple, le stress lié au terrain qui vient souvent altérer la prise de recul et l'analyse pertinente de l'action à mener en situation d'urgence ne peut être reproduit par les outils de simulation virtuelle. À l'inverse, à force de s'entrainer à partir de situations virtuelles, cela pourrait occasionner chez certains apprenants un excès de confiance en soi, induisant de lourdes conséquences sur les décisions prises en situation réelle. Par ailleurs, le coût humain et matériel de ce type de formations reste élevé et peut constituer un frein pour promouvoir leur accès.

En ce qui concerne la culture de radioprotection à destination du grand public, les participants estiment qu'il s'agit là d'un enjeu important pour les années à venir. En effet, la question du nucléaire et de la radioactivité - difficile à appréhender de prime abord - a tendance à véhiculer de «fausses idées » et des "préjugés ». Il semble ainsi important, aux yeux des participants, que le domaine de la radioprotection cherche à davantage sensibiliser sur le risque nucléaire. Il s'agirait alors de veiller à ne pas avoir exclusivement un discours scientifique et technique, mais à s'ouvrir à d'autres disciplines telles que les sciences sociales, aidant à aborder

\footnotetext{
${ }^{4}$ Futurs «Conseillers en Radioprotection», d'après le Décret $\mathrm{n}^{\mathrm{o}} 2018-437 \mathrm{du} 4$ juin 2018 relatif à la radioprotection des travailleurs contre les risques dus aux rayonnements ionisants.
}

différemment la question de la perception des risques. En termes de pistes de travail, des nouveaux outils ludiques et pédagogiques (Serious Game, MOOC, etc.) ont été cités comme exemples possibles à utiliser pour sensibiliser le public sur les questions associées au domaine nucléaire. Les discussions ont également fait ressortir l'intérêt de mieux informer sur les différents types d'expositions (naturel, artificiel, médical, etc.) ainsi que sur la signification des différentes unités utilisées en radioprotection et leurs échelles de valeurs respectives. Il a d'ailleurs été proposé de travailler, en concertation avec des représentants de la population, pour construire une échelle des doses de rayonnements et de leurs risques associés, compréhensible par tous. La possibilité de créer des MOOC ou autres moyens d'information (podcasts, articles, etc.) dédiés à la question de la radioprotection et de son application à différentes situations (milieu médical, situation post-accidentelle, etc.) a été évoquée. En lien avec le retour d'expérience post-Fukushima, il serait ainsi question de diffuser largement des informations pertinentes sur la radioprotection tout en essayant de répondre aux attentes et questionnements de la population (Lochard et al., 2019). Pour cela, une démarche collaborative avec des représentants de la société civile pourrait être développée. Il s'agirait alors de définir avec eux les thématiques sur lesquelles travailler, et voir dans quelle mesure, ils pourraient être eux-mêmes impliqués dans la réalisation et la diffusion de ces outils d'information. Les résultats de projets de recherche européens actuels tels que le projet $\mathrm{ENGAGE}^{5}$, visant à étudier les processus permettant de renforcer la culture de radioprotection et l'engagement des parties prenantes, seront à prendre en compte dans ces réflexions. Les participants se sont également interrogés sur la gouvernance possible de tels projets de diffusion d'une culture pratique de radioprotection (e.g. qui est légitime pour monter ces projets? pour les mettre en œuvre? quelles parties prenantes impliquer? etc.) ainsi que sur leurs pérennités dans le temps (e.g. quelle disponibilité des acteurs pour proposer des nouveaux podcasts / MOOC périodiquement ? comment assurer le renouvellement des personnes impliquées? etc.).

Pour finir les discussions de cet atelier ont soulevé l'importance de la sensibilisation aux enjeux de radioprotection auprès des élèves et étudiants. La nécessité de former des facilitateurs locaux (e.g. médecins, pharmaciens, élus locaux, etc.) qui - en cas d'accident nucléaire - seraient les premiers à répondre aux questionnements de la population a également été évoquée. À ce jour, plusieurs travaux en cours abordent ce sujet (e.g. TERRITORIES ${ }^{6}$, CONFIDENCE ${ }^{7}$, SHAMISEN $^{8}$, GT Santé CODIRPA) et mériteraient donc

\footnotetext{
${ }^{5}$ ENhancinG stAkeholder participation in the GovernancE of radiological risks - www.engage-concert.eu.

${ }^{6}$ To Enhance unceRtainties Reduction and stakeholders Involvement TOwards integrated and graded Risk management of humans and wildlife In long-lasting radiological Exposure Situations - territories. eu.

${ }^{7}$ Coping with uncertainties for improved modelling and decision making in nuclear emergencies - portal.iket.kit.edu/CONFIDENCE/ index.php.

${ }^{8}$ Nuclear Emergency Situations Improvement of Medical and Health Surveillance - http://radiation.isglobal.org/index.php/en/shamisenhome.
} 
d'être intégrés dans ces réflexions. De plus, en parallèle de ces démarches de sensibilisation, certains participants ont fait valoir le besoin de formations dédiées aux experts en radioprotection pour améliorer leur capacité d'information et de communication auprès du grand public.

\subsection{L'innovation au service d'un meilleur partage des données: rendre visible l'invisible}

Les discussions au cours de ces deux ateliers ont soulevé un autre enjeu d'intérêt pour l'avenir: celui de « rendre visible l'invisible» en cherchant à «démocratiser» la mesure et la caractérisation de la dose au travers de dispositifs innovants. Les nouvelles applications mobiles permettant à tous les citoyens de mesurer le débit de dose ambiant de son environnement (e.g. OpenRadiation ${ }^{9}$ (Bottollier-Depois et al., 2017), Safecast ${ }^{10}$ (Brown et al., 2016) ont ainsi été citées en exemple). Le principe de l'OpenData permettant d'accéder à des données produites par les différents utilisateurs de ces applications mobiles (e.g. citoyens) a été aussi mis en avant comme outil de partage des données pouvant être utiles tant pour les professionnels de la radioprotection, que pour le grand public. Les travaux du projet de recherche européen SHAMISEN-SINGS ${ }^{11}$, proposant une analyse des nouvelles applications mobiles dédiées à la mesure de la radioactivité, seront à mettre en regard de ces réflexions.

D'un point de vue de l'exposition professionnelle, la maîtrise du risque d'exposition aux rayonnements ionisants passe par le contrôle de la dose que chaque travailleur pourrait être susceptible de recevoir ainsi que la maitrise des effets potentiels que cela pourrait engendrer sur sa santé. La démocratisation de l'accès à cette information pourrait être réalisée en donnant la possibilité à chaque travailleur de mesurer sa dose via des applications mobiles. Cet accès à l'information pourrait également se faire en ayant accès en temps réel à la base de données SISERI ${ }^{12}$ afin de connaître l'exposition individuelle de chacun. Cela reviendrait en quelque sorte à fonctionner selon le principe de l'OpenData pour les mesures de doses en radioprotection. Toujours en lien avec la question des expositions professionnelles, les travaux menés dans le cadre du projet de recherche européen PODIUM $^{13}$ - visant à améliorer la dosimétrie professionnelle en développant une approche innovante de calcul à partir d'une application de dosimétrie en ligne basée sur des simulations informatiques, sans utiliser de dosimètres physiques - pourront également présenter des enjeux pour l'avenir. Â noter que les participants se sont également demandés pourquoi la dose liée à l'exposition médicale voire naturelle n'est-elle pas prise en compte avec la dose susceptible d'être reçue lors d'une activité

\footnotetext{
$\overline{{ }^{9} \text { www.openradiation.org/fr. }}$

${ }^{10}$ blog.safecast.org/.

${ }^{11}$ Nuclear Emergency Situations Improvement of Medical And Health Surveillance - Stakeholder Involvement in Generating Science after Nuclear Emergencies - http://radiation.isglobal.org/ index.php/en/shamisen-sings-home.

${ }^{12}$ Système d'Information de la Surveillance de l'Exposition aux Rayonnements Ionisants - https://siseri.irsn.fr/.

${ }^{13}$ Personal OnlIne Dosimetry using Computatinal Methods podium-concerth2020.eu.
}

professionnelle soumise aux rayonnements ionisants. Cela permettrait selon eux de mieux refléter la réalité d'un point de vue des effets potentiels sur le corps humain.

Les participants ont également mis en avant la possibilité de créer une base de données recensant les expositions individuelles reçues lors d'examens médicaux tout au long de la vie des patients. D'après eux, ce type de base de données permettrait en effet d'obtenir un suivi précis pour chaque patient et, ainsi d'optimiser la prescription d'examens médicaux. Sur cette base, les participants se sont d'ailleurs montrés favorables à la mise en place d'un dossier médical dématérialisé qui pourrait être consultable d'un praticien à l'autre au moyen de la carte vitale du patient. Un dispositif de contrôle pourrait par exemple être mis en place et viserait à alerter le praticien dans le cas où les doses reçues par un patient seraient trop importantes.

Un autre intérêt de la base de données pourrait être également d'obtenir des informations supplémentaires quant aux examens médicaux réalisés par les salariés du nucléaire en dehors de ceux effectués dans le cadre du suivi individuel renforcé. Cela permettrait ainsi au médecin du travail d'avoir une vision complète de l'ensemble des doses reçues par le salarié. À noter que les participants à cet atelier de réflexion n'ont pas manqué de souligner les écueils possibles associés à une telle base de données qui, en termes éthiques, suppose de veiller à bien prendre en compte la protection des données personnelles et à respecter le secret médical.

Enfin, la diffusion des données environnementales collectées par les institutionnels, selon le principe de l'OpenData, peut également être un moyen d'engager le dialogue avec la population. L'initiative du Réseau National de Mesure de la radioactivité de l'environnement ${ }^{14}(\mathrm{RNM})$, piloté par l'ASN et l'IRSN, s'inscrit déjà dans cette perspective et il pourrait être intéressant, selon les participants, de voir dans quelle mesure cet outil pourrait être davantage valorisé en y intégrant par exemple des données produites par les citoyens eux-mêmes.

Cependant, l'utilisation du principe de l'OpenData peut susciter un certain nombre de questions : peut-on par exemple, d'un point de vue éthique, autoriser un accès aux bases de données sans aucune restriction? Quelle entité serait légitime pour collecter l'ensemble des données ainsi que pour en assurer l'analyse ? Et, dans une approche holistique, comment intégrer et évaluer les autres risques en plus du risque d'exposition aux rayonnements ionisants (risques chimique, biologique, etc.)?

\section{Conclusion}

La première journée scientifique du Club des Jeunes Sociétaires de la SFRP a rassemblé plus d'une cinquantaine de participants pour la plupart étudiants ou jeunes professionnels. Les différentes présentations proposées le matin ont permis aux participants d'aborder plusieurs thématiques liées à l'innovation en radioprotection en traitant de nombreux domaines tels que la radioprotection des patients, des travailleurs ou encore la gestion des situations accidentelles et post-accidentelles. Les participants ont montré un grand

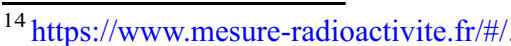


intérêt pour tous les sujets présentés qu'ils n'ont d'ailleurs pas manqué de citer en exemple lors de leurs interventions au cours des ateliers de réflexion organisés l'après-midi.

Les échanges et les contributions des participants aux deux ateliers de réflexion ont permis de faire ressortir différents enjeux d'intérêt pour l'avenir de la radioprotection au service du public et des travailleurs. Il s'agit de l'innovation pour la promotion d'une culture pratique de radioprotection, ainsi que l'innovation pour un meilleur partage des données. Pour ces deux enjeux, différentes pistes de travail ont été proposées et le Club des Jeunes Sociétaires pourrait - pour certaines - tenter de les mettre en œuvre, en collaboration possible avec les autres sections ou commissions de la SFRP (section environnement, recherche et santé, commission enseignement, etc.). À titre d'exemple, le Club des Jeunes Sociétaires pourrait s'impliquer sur la mise en place d'actions de sensibilisation sur les enjeux de radioprotection auprès des élèves et étudiants. Aussi, des journées en collaboration avec certaines sections de la SFRP pourraient être organisées sur certains challenges soulevés par cette première journée (la question de l'OpenData notamment).

À noter que d'autres enjeux plus transverses sont également ressortis lors de cette première journée scientifique, à commencer par la question de la gestion holistique du risque, ainsi que la nécessaire prise en compte des enjeux éthiques associés à la radioprotection.

Dans le prolongement de cette première journée, le Club des Jeunes Sociétaires a organisé le 26 mars 2019 une soirée «afterwork»sur le thème de l'éthique en radioprotection. Cette soirée a permis à des étudiants et des jeunes professionnels d'échanger ensemble sur ces aspects en présence de deux membres de la CIPR ayant notamment contribué à la publication 138 dédiée aux fondements éthiques du système de radioprotection. Cette soirée fut ainsi l'occasion d'échanger sur la culture de radioprotection au sein d'un groupe de jeunes professionnels, action ciblée lors des discussions de la première journée scientifique.

En termes de perspectives, l'enquête réalisée à l'issue de cette première journée scientifique met en avant la satisfaction générale de l'ensemble des participants qui encouragent de manière unanime le Club des Jeunes Sociétaires à poursuivre ce genre d'actions, avec notamment l'organisation de journées scientifiques tous les deux ans. À ce titre, la prochaine journée scientifique du Club des Jeunes Sociétaires se tiendra dans le courant du premier semestre 2020. Il pourra être ainsi intéressant de s'appuyer sur les conclusions de cette première journée pour lancer de nouveaux ateliers d'échanges d'information et de points de vue.

\section{Références}

Bottollier-Depois JF, Allain E, Baumont G, Berthelot N, Clairand I, Couvez C, Darley G, Henry B, Jolivet T, Laroche P, Lebau-Livé A, Lejeune V, Miss J, Monange W, Quéinnec F, Richet Y, Simon C, Trompier F, Vayron F. 2017. OpenRadiation: A collaborative project for radioactivity measurement in the environment by the public. Eur. Phys. J. Conf. 153: 08002.

Bourguignon M, Bérard P, Bertho JM, Farah J, Mercat C. 2017. Radioprotection: quel avenir ? Radioprotection 52(1): 13-20.

Brown A, Franken P, Bonner S, Dolezal N, Moross J. 2016. Safecast: Successful citizen-science for radiation measurement and communication after Fukushima. J. Radiol. Prot. 36: S82-S101.

Lochard J, Schneider T, Ando R, Niwa O, Clement C, Lecomte JF, Tada JI. 2019. An overview of the dialogue meetings initiated by ICRP in Japan after the Fukushima accident. Radioprotection 54 (2): $87-101$.

Citation de l'article : Ménard V, Maître M, Chambrette M, Ayadi B-M, Beaumont T, Beltrami L-A. 2019. Radioprotection: Quelles innovations? Quel avenir? Retour sur la première journée scientifique du Club des Jeunes Sociétaires de la SFRP. Radioprotection 54(4): $271-275$ 